\title{
Croissance osseuse chez le foetus ovin
}

\author{
H Collignon, MJ Davicco, P Lebecque, JP Barlet \\ INRA, Croissance et Métabolisme des Herbivores, Theix, 63122 St-Genès-Champanelle, France
}

Chez certaines espèces domestiques prolifiques (moutons, porcs) une part importante $(30 \%)$ des jeunes issus de portées multiples présente une insuffisance pondérale à la naissance (hypotrophie). Dans l'espèce ovine, $Y$ Houssin-Villette et $M$ Thériez (1981, Ann Zootech, 30, 169-182) ont montré que les agneaux hypotrophiques alimentés normalement étaient incapables de manifester une croissance compensatrice et produisaient des carcasses trop grasses, difficiles à valoriser. Cette hypotrophie se caractérise notamment par un développement insuffisant du squelette à la naissance confirmé par le dosage de l'ostéocalcine plasmatique, qui est un marqueur dynamique de la formation osseuse. En effet, les agneaux hypotrophes (poids $<2,5 \mathrm{~kg}$ ) ont à la naissance une ostéocalcinémie inférieure à celle des agneaux de grande taille (poids $>4,5 \mathrm{~kg}$ ), cette différence disparaissant quelques jours après la naissance.

Afin de préciser le stade foetal où apparaît ce ralentissement de la croissance osseuse, nous avons mesuré et déterminé la composition en minéraux de l'os canon, et dosé l'ostéocalcine dans le plasma. Les 50 foetus ovins de génotype (Limousin x Romanov) x (lle-deFrance) utilisés sont issus de portées multiples, et âgés de 75 à 145 jours (durée de gestation). Les os canons sont prélevés et disséqués pour mesurer la longueur et la largeur de la diaphyse. Le poids en matière sèche de la diaphyse est déterminé après $48 \mathrm{~h}$ à $100^{\circ} \mathrm{C}$ et le poids en matière minérale après $16 \mathrm{~h}$ d'incinération à $600^{\circ} \mathrm{C}$. Le calcium est dosé par spectrophotométrie d'absorption atomique, le phosphore par colorimétrie (méthode de Misson) et l'ostéocalcinémie est mesurée par dosage radio-immunologique (Incstar).

Le poids foetal $(\mathrm{kg})$ augmente régulièrement entre le $85^{\circ}(0,37)$ et le 132 e jour de gestation $(3,05)$; à la naissance, les poids moyens des agneaux issus de portées triples et quadruples sont respectivement de 3,34 et de 2,69 . La croissance $(\mathrm{mm} / \mathrm{jour})$ en longueur $(0,82)$ et en largeur $(0,09)$ de l'os canon est linéaire entre le $75 \mathrm{e}$ jour et la naissance. La matière sèche (MS), les matières minérales, le contenu en calcium et phosphore ont une évolution parallèle : une faible augmentation de la MS entre le $7^{\circ}(0,09 \mathrm{~g})$ et le $89^{\mathrm{e}}$ jour $(0,19 \mathrm{~g})$ suivie d'une plus forte entre le $89^{\mathrm{e}}$ et le $132^{\mathrm{e}}$ jour $(2,87 \pm 0,14 \mathrm{~g})$ puis d'une stabilisation jusqu'à la naissance (MS $=3,20 \pm 0,21 \mathrm{~g}$ ). L'évolution parallèle de ces paramètres est confirmée par des corrélations linéaires supérieures à 0,993 : en moyenne, la matière minérale représentant $61 \%$ de la MS, contient $36 \%$ de calcium et $18 \%$ de phosphore, le rapport $\mathrm{Ca} / \mathrm{P}$ est constant à 2,03 . Enfin, l'ostéocalcinémie (ng/ml) qui est à 45 au $85^{\mathrm{e}}$ jour, augmente jusqu'au $132^{e}$ jour (231) et diminue à la naissance (117).

Chez les foetus issus de portées multiples ( 3 à 4), le poids à la naissance semble être atteint au $132^{e}$ jour de gestation, stade à partir duquel les poids en matière sèche, matière minérale, calcium et phosphore n'évoluent plus. Le ralentissement de la formation osseuse après 132 jours est confirmé par la diminution de l'ostéocalcinémie. 